\title{
Luteal phase support for in vitro fertilization/intracytoplasmic sperm injection fresh cycles: a systematic review and network meta-analysis
}

\author{
Hanglin Wu', Songying Zhang ${ }^{2}$, Xiaona Lin², Shasha Wang ${ }^{2}$ and Ping Zhou ${ }^{2^{*}}$ (D)
}

\begin{abstract}
Background: Various luteal phase supports (LPSs) have been proven to increase the pregnancy rate in fresh cycles of in vitro fertilization or intracytoplasmic sperm injection; however, there is still significant debate regarding the optimal use of LPS.

Methods: A systematic review with the use of a network meta-analysis was performed via electronic searching of Ovid MEDLINE, the Cochrane Library, Embase, Web of Science, ClinicalTrials.gov and Google Scholar (up to January 2021) to compare the effectiveness and safety of various LPSs, as well as to evaluate the effects of different initiations of LPSs on pregnancy outcomes. The primary outcomes included live birth and ongoing pregnancy, with the results presented as odds ratios (ORs) with 95\% confidence intervals (Cls).

Results: Eighty-nine randomized controlled trials with 29,625 women comparing 14 interventions or placebo/no LPS treatments were included in the meta-analyses. No significant differences were found in terms of the pregnancy outcomes when LPS was started within $48 \mathrm{~h}$ after oocyte retrieval versus a delayed initiation between $48 \mathrm{~h}$ and $96 \mathrm{~h}$ after oocyte retrieval. The addition of gonadotropin-releasing hormone $(\mathrm{GnRH})$ agonists to progesterone vaginal pessaries showed a significant benefit in terms of live birth (OR 1.39, $95 \% \mathrm{Cl} 1.08$ to 1.78). Only human chorionic gonadotropin (HCG) was found to be more efficacious than the placebo/no LPS treatment in terms of live birth (OR $15.43,95 \% \mathrm{Cl} 2.03$ to 117.12 , low evidence). Any active LPSs (except for rectal or subcutaneous progesterone) was significantly more efficacious than the placebo/no LPS treatment in terms of ongoing pregnancy, with ORs ranging between 1.77 (95\% Cl 1.08 to 2.90) for the vaginal progesterone pessary and 2.14 (1.23 to 3.70) for the intramuscular progesterone treatment. Among the comparisons of efficacy and tolerability between the active treatments, the differences were small and very uncertain.
\end{abstract}

\footnotetext{
* Correspondence: zhouping0606@zju.edu.cn

${ }^{2}$ Assisted Reproduction Unit, Department of Obstetrics and Gynecology, Sir

Run Run Shaw Hospital, Zhejiang University School of Medicine, No. 3

Qingchun East Road, Hangzhou 310016, China

Full list of author information is available at the end of the article
}

(C) The Author(s). 2021 Open Access This article is licensed under a Creative Commons Attribution 4.0 International License, which permits use, sharing, adaptation, distribution and reproduction in any medium or format, as long as you give appropriate credit to the original author(s) and the source, provide a link to the Creative Commons licence, and indicate if changes were made. The images or other third party material in this article are included in the article's Creative Commons licence, unless indicated otherwise in a credit line to the material. If material is not included in the article's Creative Commons licence and your intended use is not permitted by statutory regulation or exceeds the permitted use, you will need to obtain permission directly from the copyright holder. To view a copy of this licence, visit http://creativecommons.org/licenses/by/4.0/. The Creative Commons Public Domain Dedication waiver (http://creativecommons.org/publicdomain/zero/1.0/) applies to the data made available in this article, unless otherwise stated in a credit line to the data. 
Conclusion: Delays in progesterone supplementation until $96 \mathrm{~h}$ after oocyte retrieval does not affect pregnancy outcomes. The safety of GnRH agonists during the luteal phase needs to be evaluated in future studies before the applications of these agonists in clinical practice. With comparable efficacy and acceptability, there may be several viable clinical options for LPS.

Keywords: In vitro fertilization, Intracytoplasmic sperm injection, Luteal phase supports, Pregnancy rate, Network metaanalysis

\section{Introduction}

Assisted reproductive technology (ART) is a widely accepted procedure for couples seeking fertility aid due to infertility, with more than 1 million cycles performed every year throughout the world [1]. Supraphysiological levels of steroids that result from controlled ovarian stimulation (COS) in ART cycles are thought to inhibit pituitary luteinizing hormone secretion, thus shortening the luteal phase, which consequently causes luteal phase deficiency $[2,3]$. To overcome this issue, various exogenous luteal phase supports (LPSs), including progesterone, human chorionic gonadotropin (HCG), oestrogen, gonadotropin-releasing hormone $(\mathrm{GnRH})$ agonists or combinations of several of these support types, have been used to compensate for the progesterone levels; however, there is still significant debate regarding optimal LPS use.

A Cochrane review in 2015 evaluated the relative effectiveness and safety of the available methods of LPS for women undergoing ART, and the authors found no conclusive evidence regarding progesterone, HCG or the addition of oestrogen [3]. Due to the fact that some studies were not included [4-15], as well as the fact that many new randomized controlled trials (RCTs) have recently been published [16-31], the performance of a current, updated systematic review and meta-analysis at this time is warranted. Recently, several meta-analyses have examined the efficacies of oral progesterone, subcutaneous progesterone and $\mathrm{GnRH}$ agonists that are used during the luteal phase [32-35]. These approaches provided limited insights into the treatment hierarchy which made it difficult for clinicians to choose an optimal LPS; therefore, it is necessary to synthesize both direct and indirect available evidence from existing trials to compare the relative effects of multiple treatment options.

With regard to the timing of progesterone initiation, a systematic review recommended the initiation of LPS between the evening of oocyte retrieval and 3 days after oocyte retrieval; however, the article included limited studies that exhibited with obvious heterogeneity [36]. A recent RCT found a significantly lower ongoing pregnancy rate when starting LPS on the oocyte retrieval day, compared with day five after embryo transfer (ET) [19]. When considering the potential benefits of delaying vaginal progesterone and the shortage of meta-analyses, it would be essential to compare different progesterone initiations during the luteal phase.
This study aimed to perform a systematic review and network meta-analysis by comparing multiple LPS treatments for women with fresh cycles of in vitro fertilization (IVF) or intracytoplasmic sperm injection (ICSI), in order to inform clinical practice. Furthermore, we evaluated the effects of different initiations of LPS on the pregnancy outcomes.

\section{Methods}

Search strategy and selection criteria

We searched Ovid MEDLINE, the Cochrane Library, Embase, Web of Science, ClinicalTrials.gov and Google Scholar for RCTs published from the date of database inception to October 27th, 2018 and updated the search on January 9th, 2021. No language limit was applied. We used MeSH headings "fertilization in vitro", "sperm injections, intracytoplasmic", "ET", "luteal phase", "progesterone", "chorionic gonadotropin", "chorionic gonadotropin, beta subunit, human", "gonadotropin-releasing hormone", "oestrogens", "estradiol", and combined them with text words and word variants. The reference lists of selected articles and reviews were hand searched to identify any relevant articles. Study authors were contacted to supplement incomplete reports of the original papers. Detailed search strategy for Ovid MEDLINE can be found in Appendix S1.

\section{Study selection}

We followed the PRISMA guidelines for network metaanalysis [37]. The study protocol was registered with PROSPERO (CRD42018115011). We selected studies for inclusion in two stages. Conference abstracts, duplicates or irrelevant articles were excluded by screening the titles and abstracts, and all of the remaining articles were screened via their full texts. Two authors independently did the screening and assessed them for eligibility with discrepancies resolved with an additional reviewer. We included RCTs that compared any agent that was used for LPS in a IVF/ICSI fresh cycle. We excluded crossover trials and quasi-RCTs. We also excluded studies involving intrauterine insemination, gamete intrafallopian transfer, zygote intrafallopian transfer and embryo transfer from donated oocytes or in vitro maturation cycles.

The participants included subfertile women undergoing IVF/ICSI fresh cycles for any reason. For inclusion, 
at least 10 women were required in any intervention or control group in the studies. Studies were excluded for the preservation of homogeneity if inconsistent procedures (such as different COS) were performed before oocyte retrieval in the groups. Studies including GnRH or dual triggering were also excluded. Studies had to include at least two of the following LPS categories: "any type, dose or route of progesterone which had to be provided at least five doses or continued beyond positive pregnancy test", "any type, dose or route of HCG which had to be provided at least two doses", "aforementioned progesterone with any type, dose or route of HCG", "aforementioned progesterone with any type, dose or route of GnRH agonists", "aforementioned progesterone with any type, dose or route of oestrogen", "other interventions found" and "placebo or no LPS treatment". Complex or rarely used LPSs were excluded.

\section{Study quality assessment and data extraction}

Two independent reviewers undertook study quality assessment and data extraction. Any discrepancies were resolved by discussion within the review team. We assessed the risk of bias according to the Cochrane Handbook for Systematic Reviews of Interventions [38]. Specifically, attention was focused on seven domains, i.e., random sequence generation, allocation concealment, blinding of participants and personnel, blinding of outcome assessment, incomplete outcome data, selective reporting and other biases. The reviews categorized studies as "low risk", "high risk" or "unclear risk" of bias.

Relevant information from the included trials was extracted with a predefined data extraction sheet. The extracted data included study characteristics (including published year, country and study sample size), patient characteristics (eligibility criteria), procedures (COS protocol, criteria of triggering, ovulation triggering, day of oocyte retrieval, fertilization, day of ET and ET policy), interventions (initiation, protocol and duration of LPS) and outcomes (live birth, clinical pregnancy, ongoing pregnancy, miscarriage and adverse effects).

We considered live birth and ongoing pregnancy for our primary analyses. Live birth was defined as the delivery of one or more living infants. Ongoing pregnancy was defined as a pregnancy beyond 12 weeks' gestation. Our secondary outcomes included clinical pregnancy (defined as the presence of a gestational sac, with or without a fetal heartbeat on ultrasonography), miscarriage (defined as pregnancy loss after confirmation of clinical pregnancy) and adverse effects (including ovarian hyperstimulation syndrome [OHSS] and other system disorders). If pregnancy was not further stated, then we regarded it as being a clinical pregnancy. In cases of certain discrepancies in the definition, we accepted the primary study authors' definition, when relevant.

\section{Data synthesis and statistical analysis}

We categorized the interventions according to the different types, routes and combinations of LPS. When considering comparable pregnancy outcomes, we combined vaginal tablets with vaginal suppositories, and an aggregation of the different doses of progesterone was performed [39-42]. When multiple doses of progesterone were used within a trial, a pooled amount of the data was used. The odds ratios (ORs) and risk differences were calculated for all of the outcomes with $95 \%$ confidence intervals (CIs). For network meta-analysis, we used a continuity correction for studies with no events by adding 0.5 to both the events count and the total sample size.

We did two separate analyses. First, when considering comparable pregnancy rates when beginning LPS on the evening of oocyte retrieval or 1 day later, we classified the initiation of LPS as being early (within $48 \mathrm{~h}$ after oocyte retrieval) and delayed (48-96 $\mathrm{h}$ after oocyte retrieval) [18]. We performed a multivariate network meta-analysis to evaluate the effects of different initiations of LPS, and we speculated that there would be no significant differences.

Second, we analysed all of the interventions (regardless of initiation). We performed pairwise meta-analyses of the pregnancy outcomes with the random-effects model (the Mantel-Haenszel method). We assessed statistical heterogeneity in each pairwise comparison with the $\mathrm{I}^{2}$ statistic and $p$ value [43]. To visualize the network geometry and node connectivity, we produced network plots for the outcomes [44]. Afterwards, we performed a network meta-analysis by using the methodology of the multivariate meta-analysis model. We prepared league tables presenting mixed comparisons for the inspections of both types of evidence [45]. We estimated the ranking probabilities for all of the treatments of being at each possible rank for each intervention and the treatment hierarchy was summarized as the surface under the cumulative ranking curve [46].

The assessment of statistical heterogeneity in the networks was based on the magnitude of the heterogeneity variance parameter ( $\tau 2)$ estimated from the network meta-analysis models. To check the assumption of consistency in the entire network, we used the designby-treatment model and judged the presence of inconsistency based on a $\mathrm{Chi}^{2}$ test [47]. Local inconsistency between direct and indirect sources of evidence was statistically assessed by calculation of the difference between direct and indirect estimates in all closed loops in the network [44].

To evaluate the presence of small study effects, we visually inspected the comparison-adjusted funnel plots for the pregnancy outcomes [44]. We conducted sensitivity analyses to assess the robustness of our findings by 
excluding trials that were published before 2010. We prepared analyses of the data in the following subgroups (if enough studies were available): 1 . different COS protocols; 2 . participants with previously failed cycles, including $\leq$ two failed ART cycles and $>$ two failed ART cycles; and 3. the number of embryos transferred, including single or more than one transferred embryos. The certainty of the evidence produced by the synthesis for each outcome was evaluated by using the GRADE approach and the framework described by Salanti and colleagues $[46,48]$. Statistical analyses were performed with the use of STATA (version 14.0).

\section{Results}

Overall, 4791 citations were identified with the search and 175 potentially eligible articles were retrieved in the full text. We excluded 81 reports but included two additional studies after reviewing the reference lists, thus resulting in 96 publications that were published between 1987 and
2020 (Fig. 1 and Table S1, full references for all of the trials are provided in Appendix S2). Seven studies were excluded from the meta-analysis for various reasons (Appendix S2).

Overall, 89 RCTs with 29,625 women comparing 14 interventions or placebo/no LPS treatments were included in the meta-analysis. Information concerning the risk of bias was obtained from three authors (Aghahosseini Marzieh, Bergh Christina and Nasrin Saharkhiz). Information concerning ovarian stimulation and embryo transfer was obtained from two authors (Chi HB and Monique H.Mochtar). The mean study sample size was 333 participants, ranging between 30 and 1983 patients. No study was found that involved the performance of single ET. Vaginal progesterone gels, tablets or suppositories were more commonly used than other LPSs. In terms of study quality, 52 trials were classified as exhibiting a low risk for random sequence generation. Most studies lacked the blinding of participants, personnel or outcome assessment (Figure S1 and S2).

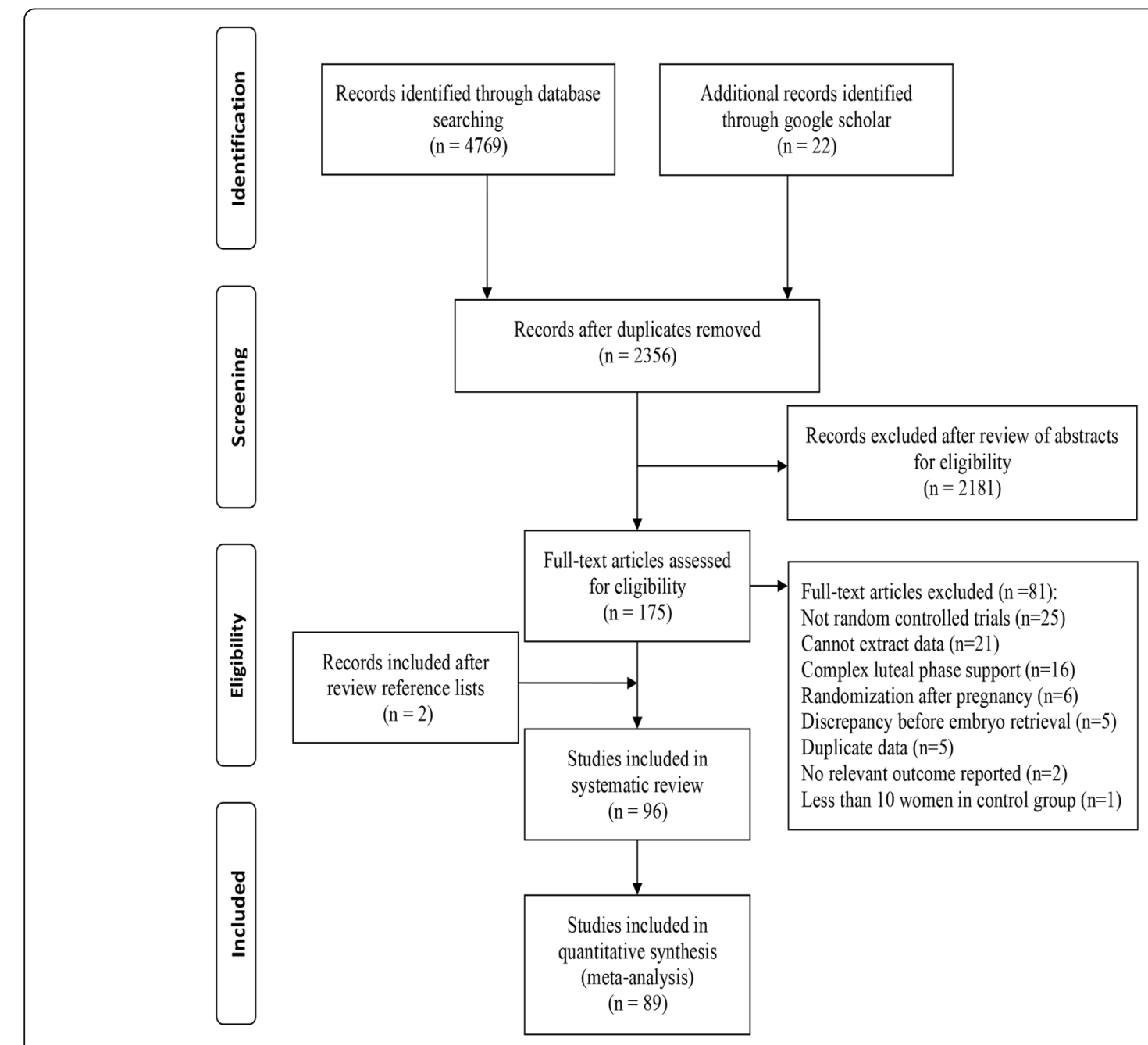

Fig. 1 Article retrieval and screening 
Table 1 shows the study and patient characteristics in terms of live birth and ongoing pregnancy. The median age of the patients was 32.1 years for ongoing pregnancy. Most trials recruited women from North America, Europe and Asia. More than half of the trials used a long $\mathrm{GnRH}$ agonist protocol. ICSI was used in approximately $50 \%$ of trails. Seventy-two percent of the trails performed LPS within $48 \mathrm{~h}$ after oocyte retrieval.

Figure 2 shows the network of eligible comparisons of LPS in different initiations for the primary outcomes. The results of the network meta-analysis for pregnancy outcomes are presented in Fig. 3 and Figure S3. The early administration of progesterone showed no evidence of an effect on live birth being greater than the delayed (ORs ranging from 0.87 for vaginal progesterone gel to 1.14 for intramuscular progesterone, with a very low degree of evidence). When compared with delayed LPS, early LPS was not associated with significantly higher ongoing pregnancy rates, with estimated ORs ranging from 0.82 (95\% CI 0.23 to 2.87) for rectal progesterone to 4.52 (95\% CI 0.17 to 118.02 ) for HCG. The evidence was either low or very low for these results. No significant differences were found in any of the comparisons in terms of clinical pregnancy and miscarriage. A fitting of the design-by-treatment interaction model provided no evidence for statistically significant inconsistency for pregnancy outcomes $(P=0.11$ to 0.68$)$. We found no significant evidence for local inconsistency in all of the closed loops (Figure S4).

Figure 4 shows the network of eligible comparisons of LPS for primary outcomes. Only HCG was found to be more efficacious than placebo/no LPS treatment in

Table 1 General characteristics of studies. Values are numbers (percentages) unless stated otherwise

\begin{tabular}{|c|c|c|}
\hline Characteristics & Live birth $(n=25)$ & Ongoing pregnancy $(n=43)$ \\
\hline \multicolumn{3}{|l|}{ Study characteristics } \\
\hline Median (range) study sample size & $355(38-1983)$ & $237(38-1983)$ \\
\hline \multicolumn{3}{|l|}{ Continent } \\
\hline North America & $5(20)$ & $9(21)$ \\
\hline Europe & $9(36)$ & $17(40)$ \\
\hline Asia & $7(28)$ & $11(26)$ \\
\hline World wide & $2(8)$ & $2(5)$ \\
\hline Other & $2(8)$ & $4(9)$ \\
\hline \multicolumn{3}{|l|}{ Type of interventions/controls } \\
\hline Placebo/no LPS treatment & $2(8)$ & $6(14)$ \\
\hline HCG & $2(8)$ & $5(12)$ \\
\hline Progesterone & $24(96)$ & $39(91)$ \\
\hline Combined $^{a}$ & $6(24)$ & $11(26)$ \\
\hline \multicolumn{3}{|l|}{ Patient characteristics } \\
\hline Median (range) age (years); No in group & $32.4(28.4-35.5) ; n=24$ & $32.1(28.4-35.4) ; n=40$ \\
\hline \multicolumn{3}{|l|}{ Ovarian stimulation } \\
\hline Long GnRH agonist protocol & $14(56)$ & $24(56)$ \\
\hline GnRH antagonist protocol & $1(4)$ & $5(12)$ \\
\hline Other protocol & $4(16)$ & $6(14)$ \\
\hline Combined & $6(24)$ & $8(19)$ \\
\hline \multicolumn{3}{|l|}{ Fertilization } \\
\hline IVF & $13(52)$ & $23(53)$ \\
\hline ICSI & $2(8)$ & $7(16)$ \\
\hline IVF/ICSI & $10(40)$ & $13(30)$ \\
\hline \multicolumn{3}{|l|}{ Timing LPS after oocyte retrieval } \\
\hline Within $48 \mathrm{~h}$ & $18(72)$ & $31(72)$ \\
\hline $48-96 h$ & $4(16)$ & $12(28)$ \\
\hline Not stated & $5(20)$ & $5(12)$ \\
\hline
\end{tabular}

${ }^{a}$ Combined progesterone with HCG, oestrogen or GnRH agonists. Abbreviations LPS luteal phase support, HCG human chorionic gonadotrophin, GnRH gonadotropin releasing hormone, IVF in vitro fertilization, ICSI intracytoplasmic sperm injection 


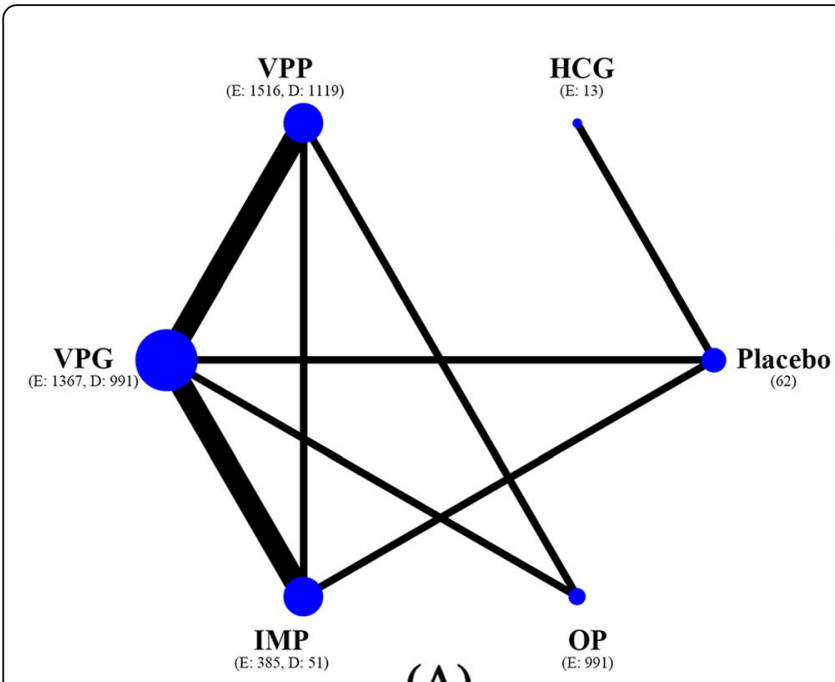

(A)

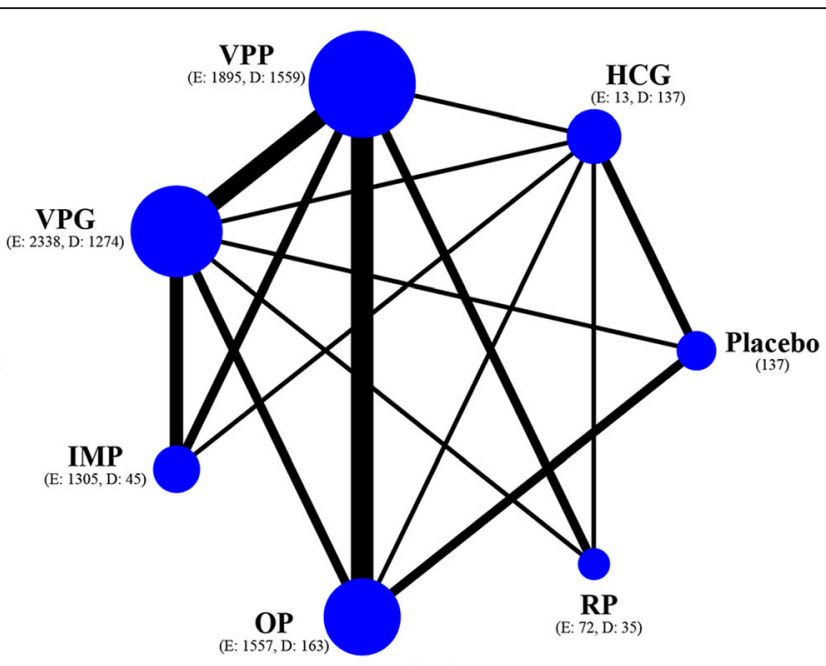

(B)

Fig. 2 Network plots of comparisons on live birth and ongoing pregnancy of different luteal phase supports in patients undergoing fresh cycles. A Live birth; B Ongoing pregnancy. Size of node is proportional to number of arms randomized to each treatment (numbers of subgroup patients with early and delayed luteal phase supports are presented in brackets). Line width is proportional to number of randomized controlled trials comparing each pair of treatments. HCG = human chorionic gonadotrophin; VPP = vaginal progesterone pessary; VPG = vaginal progesterone gel; IMP = intramuscular progesterone; $\mathrm{OP}=$ oral progesterone; $\mathrm{RP}=$ rectal progesterone

terms of live birth (OR 15.43, 95\% CI 2.03 to 117.12, low evidence, Fig. 5). Placebo treatments were significantly less efficacious than any active LPS (except for rectal or subcutaneous progesterone) in terms of ongoing pregnancy, and the ORs for LPS associated with significant improvements ranged between 1.77 (95\% CI 1.08 to 2.90) for vaginal progesterone pessary and 2.14 (95\% CI 1.23 to 3.70) for intramuscular progesterone (Fig. 5). Progesterone that was applied vaginally, orally or intramuscularly was found to be associated with a higher chance of clinical pregnancy (Figure S5). With regard to the effects of adding oestrogen, HCG or GnRH agonists in the luteal phase, the additions of oestrogen or GnRH agonists to the vaginal progesterone pessary showed a significant benefit in terms of some of the pregnancy outcomes (Figure S6). In terms of tolerability, vaginal bleeding was counted in more studies than other adverse events. There was no strong evidence of any significant difference when comparing vaginal progesterone pessary with other types (Figure S7). A network meta-analysis of OHSS was not performed, due to the absence of a closed loop. The mean rank of each treatment was plotted to

\begin{tabular}{|c|c|c|c|c|c|c|}
\hline \multirow{2}{*}{ Luteal phase support } & \multicolumn{2}{|c|}{ Live birth, n/N (\%) } & \multirow{2}{*}{\multicolumn{2}{|c|}{ Odds ratio $(95 \%$ CI) }} & \multirow{2}{*}{ Risk Difference ( $95 \%$ CI) } & \multirow{2}{*}{ Quality of evidence } \\
\hline & \multirow[t]{2}{*}{ Early } & \multirow[t]{2}{*}{ Delayed } & & & & \\
\hline & & & Direct estimate & \multicolumn{2}{|c|}{ NMA estimate } & \\
\hline Vaginal progesterone pessary & $27 / 128(21 \%)$ & $26 / 127(20 \%)$ & $1.04(0.41,2.66)$ & $1.04(0.41,2.66)$ & $0.01(-0.15,0.17)$ & Very low \\
\hline Vaginal progesterone gel & $444 / 1367(32 \%)$ & $281 / 991(28 \%)$ & . & $0.87(0.23,3.24)$ & $-0.03(-0.25,0.20)$ & Very low \\
\hline Intramuscular progesterone & $24 / 46(52 \%)$ & $25 / 51(49 \%)$ & $1.13(0.39,3.32)$ & $1.13(0.39,3.32)$ & $0.03(-0.20,0.27)$ & Very low \\
\hline \multirow{3}{*}{ Luteal phase support } & \multicolumn{2}{|c|}{ Ongoing pregnancy, n/N (\%) } & \multirow{2}{*}{\multicolumn{2}{|c|}{ Odds ratio $(95 \%$ CI) }} & \multirow{2}{*}{ Risk Difference (95\% CI) } & \multirow{2}{*}{ Quality of evidence } \\
\hline & Early & Delayed & & & & \\
\hline & & & Direct estimate & & IA estimate & \\
\hline Human chorionic gonadotrophin & $3 / 13(23 \%)$ & $48 / 137(35 \%)$ & . & $4.52(0.17,118.02)$ & $0.21(-0.09,0.52)$ & Very low \\
\hline Vaginal progesterone pessary & $647 / 1820(36 \%)$ & $467 / 1559(30 \%)$ & $0.95(0.53,1.70)$ & $0.93(0.57,1.51)$ & $-0.02(-0.11,0.08)$ & Very low \\
\hline Vaginal progesterone gel & $848 / 2338(36 \%)$ & $414 / 1274(32 \%)$ & $1.91(0.72,5.02)$ & $1.14(0.72,1.82)$ & $0.03(-0.07,0.12)$ & Very low \\
\hline Intramuscular progesterone & $33 / 86(38 \%)$ & $15 / 45(33 \%)$ & $1.25(0.58,2.67)$ & $1.25(0.58,2.67)$ & $0.05(-0.14,0.24)$ & Low \\
\hline Oral progesterone & $534 / 1557(34 \%)$ & $38 / 163(23 \%)$ & . & $1.35(0.65,2.77)$ & $0.06(-0.07,0.19)$ & Very low \\
\hline Rectal progesterone & $22 / 72(31 \%)$ & $12 / 35(34 \%)$ & . & $0.82(0.23,2.87)$ & $-0.05(-0.32,0.23)$ & Very low \\
\hline
\end{tabular}

Fig. 3 Network meta-analysis (NMA) of live birth and ongoing pregnancy according to luteal phase support initiation strategy. Abbreviations: $\mathrm{HCG}=$ human chorionic gonadotrophin; VPP = vaginal progesterone pessary; VPG = vaginal progesterone gel; IMP = intramuscular progesterone; $\mathrm{OP}=$ oral progesterone; $\mathrm{RP}=$ rectal progesterone 


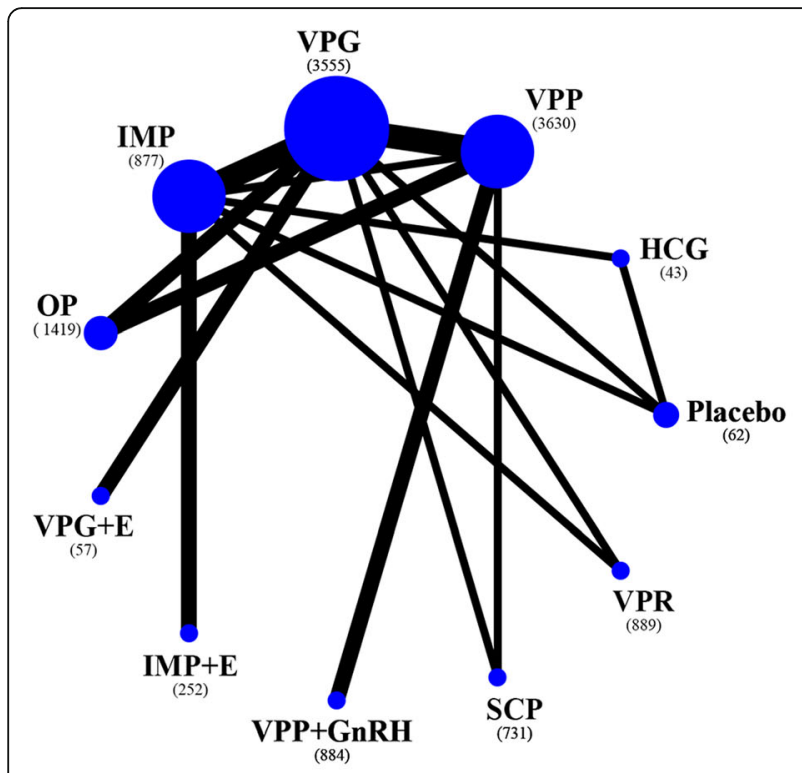

(A)

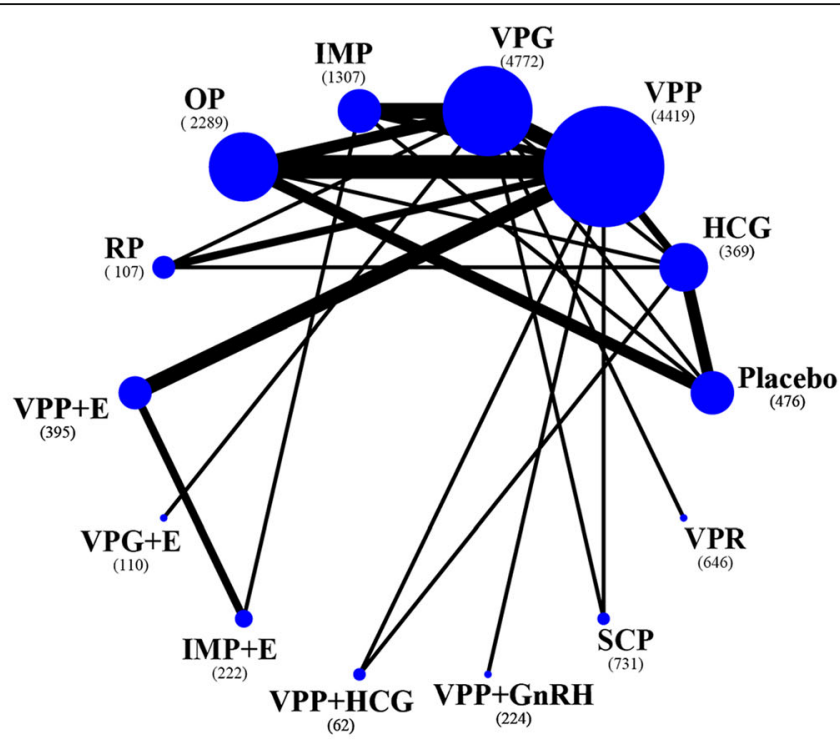

(B)

Fig. 4 Network plots of comparisons on live birth and ongoing pregnancy (regardless of the initiations of luteal phase supports). A Live birth; B Ongoing pregnancy. Size of node is proportional to number of arms randomized to each treatment (number of patients in brackets). Line width is proportional to number of randomized controlled trials comparing each pair of treatments. HCG = human chorionic gonadotrophin; VPP = vaginal progesterone pessary; $\mathrm{VPG}=$ vaginal progesterone gel; $\mathrm{IMP}=$ intramuscular progesterone; $\mathrm{OP}=$ oral progesterone; $\mathrm{RP}=$ rectal progesterone; $\mathrm{GnRH}=$ gonadotropin releasing hormone; $\mathrm{E}=$ oestrogen; $\mathrm{SCP}=$ subcutaneous progesterone; $\mathrm{VPR}=$ vaginal progesterone ring

illustrate clustering of interventions according to higher effectiveness (achieving ongoing pregnancy) and higher acceptability (reducing vaginal bleeding). Although intramuscular progesterone ranked higher on both outcomes, the probability of other adverse events was not reported in previous studies (Fig. 6).

The test of global inconsistency showed no significant difference between the consistency and inconsistency models for pregnancy outcomes $(P=0.08$ to 0.82$)$. We found no significant evidence for local inconsistency in all of the closed loops (Figure S8). Additionally, we found no strong evidence of small study effects across outcomes in the network meta-analysis (Figure S9). Predefined subgroup analyses were not available, due to the limited number of studies in some of the subgroups. Therefore, we performed a sensitivity analysis

\begin{tabular}{|c|c|c|c|c|c|c|}
\hline $\begin{array}{l}\text { Luteal phase support } \\
\text { (reference: placebo) }\end{array}$ & $\begin{array}{c}\text { No of arms } \\
\text { (placebo, } n=2)\end{array}$ & $\begin{array}{l}\text { Live birth, } \mathbf{n} / \mathbf{N}(\%) \\
\quad \text { (placebo, 2/62) }\end{array}$ & \multicolumn{2}{|c|}{ Odds ratio $(95 \% \mathrm{CI})$} & Risk Difference ( $95 \%$ CI) & Quality of evidence \\
\hline & & & Direct estimate & \multicolumn{2}{|c|}{ NMA estimate } & \\
\hline Human chorionic gonadotrophin & 2 & $9 / 43(21 \%)$ & $7.00(0.32,152.95)$ & $15.43(2.03,117.12)$ & $0.24(0.07,0.41)$ & Low \\
\hline Vaginal progesterone pessary & 9 & $1140 / 3630(31 \%)$ & . & $3.54(0.85,14.81)$ & $0.08(-0.04,0.20)$ & Very low \\
\hline Vaginal progesterone gel & 12 & $1147 / 3555(32 \%)$ & $2.08(0.37,11.91)$ & $3.45(0.83,14.34)$ & $0.07(-0.04,0.17)$ & Very low \\
\hline Intramuscular progesterone & 8 & $291 / 877(33 \%)$ & $6.71(1.41,31.99)$ & $3.93(0.96,16.15)$ & $0.10(-0.01,0.21)$ & Very low \\
\hline Oral progesterone & 4 & $446 / 1419(31 \%)$ & . & $3.93(0.93,16.50)$ & $0.09(-0.03,0.21)$ & Very low \\
\hline Subcutaneous progesterone & 2 & $252 / 731(34 \%)$ & . & $3.13(0.74,13.26)$ & $0.05(-0.09,0.18)$ & Very low \\
\hline Vaginal progesterone ring. & 2 & $369 / 889(42 \%)$ & . & $3.81(0.91,15.91)$ & $0.09(-0.03,0.22)$ & Very low \\
\hline \multirow[t]{2}{*}{$\begin{array}{l}\text { Luteal phase support } \\
\text { (reference: placebo) }\end{array}$} & $\begin{array}{c}\text { No of arms } \\
\text { (placebo, } n=6 \text { ) }\end{array}$ & $\begin{array}{c}\text { Ongoing pregnancy, n/N (\%) } \\
\text { (placebo, 47/476) }\end{array}$ & \multicolumn{2}{|c|}{ Odds ratio $(95 \% \mathrm{CI})$} & Risk Difference ( $95 \%$ CI) & Quality of evidence \\
\hline & & & Direct estimate & \multicolumn{2}{|c|}{ NMA estimate } & \\
\hline Human chorionic gonadotrophin & 5 & $72 / 369(20 \%)$ & $1.70(0.76,3.78)$ & $1.83(1.13,2.95)$ & $0.08(0.02,0.15)$ & Very low \\
\hline Vaginal progesterone pessary & 20 & $1465 / 4419(33 \%)$ & . & $1.77(1.08,2.90)$ & $0.08(0.01,0.14)$ & Very low \\
\hline Vaginal progesterone gel & 14 & $1700 / 4772(36 \%)$ & $3.26(0.63,16.97)$ & $1.87(1.13,3.09)$ & $0.09(0.03,0.15)$ & Low \\
\hline Intramuscular progesterone & 7 & $506 / 1307(39 \%)$ & $10.14(2.18,47.06)$ & $2.14(1.23,3.70)$ & $0.12(0.05,0.20)$ & Very low \\
\hline Oral progesterone & 12 & $704 / 2289(31 \%)$ & $1.39(0.73,2.65)$ & $1.79(1.11,2.89)$ & $0.08(0.02,0.14)$ & Very low \\
\hline Rectal progesterone & 2 & $34 / 107(32 \%)$ & . & $1.54(0.75,3.19)$ & $0.05(-0.09,0.18)$ & Very low \\
\hline Subcutaneous progesterone & 2 & $256 / 731(35 \%)$ & . & $1.59(0.89,2.84)$ & $0.05(-0.04,0.15)$ & Very low \\
\hline Vaginal progesterone ring. & 1 & $300 / 646(46 \%)$ & 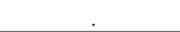 & $1.97(1.05,3.69)$ & $0.11(0.01,0.21)$ & Very low \\
\hline
\end{tabular}

Fig. 5 Network meta-analysis (NMA) for live birth and ongoing pregnancy 


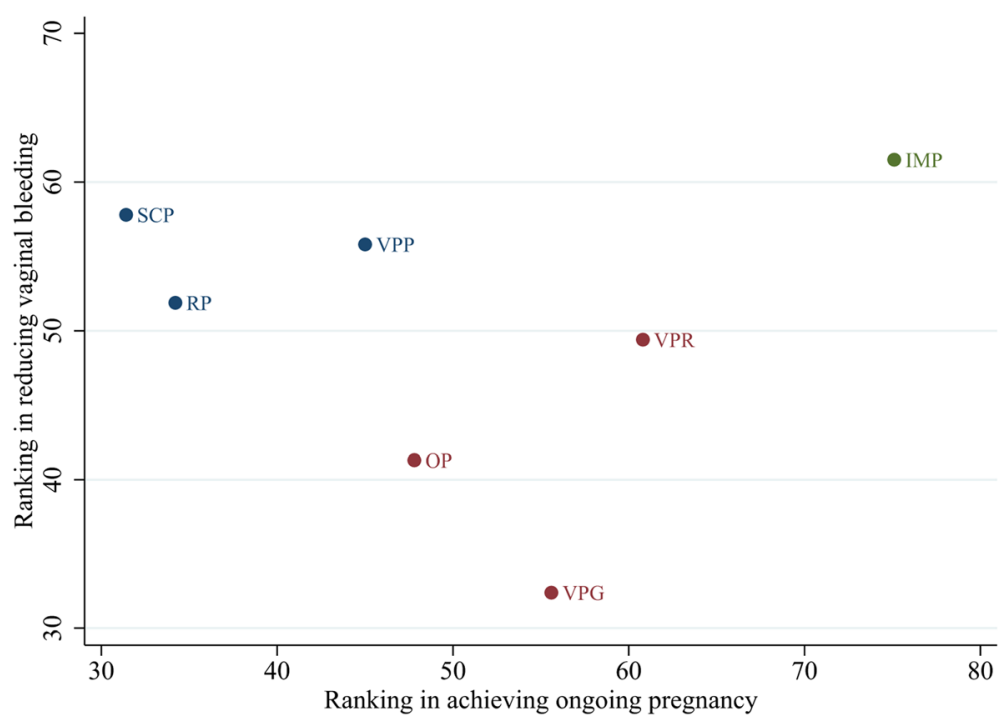

Fig. 6 Clustered ranking plot by mean rank values from results of network meta-analyses of ongoing pregnancy and vaginal bleeding. VPP =vaginal progesterone pessary; VPG = vaginal progesterone gel; $\mathrm{IMP}=$ intramuscular progesterone; $\mathrm{OP}=$ oral progesterone; $\mathrm{RP}=$ rectal progesterone; $\mathrm{SCP}=$ subcutaneous progesterone; $\mathrm{VPR}=$ vaginal progesterone ring

by including articles that used the long GnRH agonist protocol. The sensitivity analyses did not affect the main results (Figure S10).

\section{Discussion}

This analysis was based on 89 trials, which included 29, 625 couples with infertility randomly assigned to active LPS or placebo/no LPS treatments. To our knowledge, this study represents the most comprehensive synthesis of data for currently available LPSs for women undergoing IVF/ICSI. No significant differences were found in terms of all of the pregnancy outcomes when LPS was started within $48 \mathrm{~h}$ after oocyte retrieval versus delayed initiation between $48 \mathrm{~h}$ and $96 \mathrm{~h}$ after oocyte retrieval. The additions of oestrogen or HCG to regular LPS did not improve pregnancy outcomes in most cases. The administration of a GnRH agonist during the luteal phase in women undergoing IVF/ICSI was found to improve live birth and clinical pregnancy. The placebo was significantly less efficacious than any active LPS (except for rectal or subcutaneous progesterone) in terms of ongoing pregnancy and clinical pregnancy. Of the active comparisons for pregnancy and adverse outcomes, the precision of the summary treatment effect estimates varied considerably, with higher levels of uncertainty for treatments for which there were only a few available RCTs.

This was the first meta-analysis performed to compare the effects of different initiations of LPS on pregnancy outcomes, and the results were consistent with those of a previous systematic review [36]. This article did not evaluate live birth and ongoing pregnancy, which were the primary outcomes in our work. Feichtinger et al. conducted a RCT including 910 women undergoing ET 3 days after oocyte retrieval, and the group that initiated LPS 1 day after oocyte retrieval did not show any significant differences concerning pregnancy rates, compared with the group that started LPS 4 days after oocyte retrieval [49]. In this RCT, the LPS consisted of a combination of vaginal progesterone pessary, oral progesterone and oestrogen. In our study, we evaluated the different application routes of luteal phase support, which provided more evidence for the use of delayed LPS. Ghanem ME and colleagues found that the initiation of LPS on the oocyte retrieval day was associated with a poorer cycle outcome on day five (but not on day three) ET, when compared with the initiation of LPS on the ET day [19]. Williams et al. reported of a lower pregnancy rate in patients who started progesterone on day six after oocyte retrieval, compared with day three after oocyte retrieval; however, this article performed ET 3 days after oocyte retrieval [50]. In our review, only these two studies performed LPS until $96 \mathrm{~h}$ after oocyte retrieval, and we could not evaluate the influence of further delaying LPS until later timepoints $[19,50]$. Future studies are needed to address the gaps in the evidence.

Consistent with most of our results, a previous metaanalysis found no benefit of the addition of oestrogen including transdermal administrations during the luteal phase for improving IVF/ICSI outcomes [51]. Due to the limited studies and the very low evidence, the potential benefit of oestrogen that was found in our results should be interpreted with caution and needs to be evaluated in further studies. Although our results support the benefit of the addition of $\mathrm{GnRH}$ agonists during the luteal 
phase, which has been reported in several previous meta-analyses [3, 35, 52], such a benefit, if present, is unlikely to promote the application of GnRH agonists in clinical practice until their safety (including both adverse perinatal outcomes and congenital malformations) can be confirmed in future studies.

HCG seems to yield improvements in live birth rates; however, the results have mainly been based on older and smallscale studies. Concerning its higher risk of OHSS, HCG was not used in trials published after 2010 [3]. Rectal or subcutaneous progesterone seem to be less efficacious than other active LPSs; however, no significant differences were found, and the result was consistent with that of a previous analysis [33]. When considering the comparable pregnancy rates among other LPS types, we found limited evidence to recommend any LPS as being the first-line protocol for IVF/ICSI cycles. We also found a comparable miscarriage rate between the placebo and active LPS treatments, which was due to the lower clinical pregnancy rate in the control groups using the placebo. Our findings for tolerability were mainly based on limited studies, and no definite conclusion could be made at the current stage.

There were several important limitations in this study. Many of the included studies exhibited an unclear risk of bias, and most of the comparisons were assessed as being low or very low in quality, which largely restricts the interpretation of these results. Moreover, most of the studies lacked the blinding of participants, personnel or outcome assessments; nevertheless, it is unlikely that pregnancy outcomes (such as live birth or miscarriage) can be affected by a detection bias. Publication bias is always a concern, and we searched ClinicalTrials.gov and included non-English language trials. Additional information was obtained from the original authors. Some adverse events were reported in the limited number of studies, and these events were not compared in our analysis. Similarly, a cost-effectiveness analysis could not be performed in our work, which is a major concern for clinical practice.

The findings of this comprehensive network meta-analysis provide some evidence to support the late initiation of LPS, thereby reducing both costs and side effects. Herein, we provide the best available evidence suggesting that the additions of oestrogen or HCG to progesterone for LPS via different administration routes or doses do not improve the pregnancy rate. Additionally, we provide the treatment hierarchy, as well as the ORs, for pregnancy outcomes and adverse events among active uses of LPS, which can enable the personalization of clinically effective treatments without a major risk of adverse effects for women undergoing fresh IVF/ICSI cycles. The safety of adding GnRHs agonist during the luteal phase was not well studied in this population, and further research should be conducted to accumulate more evidence concerning this effect.

\section{Conclusion}

Delaying progesterone supplementation until $96 \mathrm{~h}$ after oocyte retrieval does not affect pregnancy outcomes. The current evidence does not support the addition of oestrogen or HCG during the luteal phase. The safety of GnRH agonists during the luteal phase needs to be evaluated in future studies before their application in clinical practice. There were no significant differences in the efficacy and acceptability profiles across the treatments, and the best choice may not be uniform across patients.

\section{Abbreviations \\ ART: Assisted reproductive technology; COS: Controlled ovarian stimulation; LPSs: Luteal phase supports; HCG: Human chorionic gonadotropin; GnRH: Gonadotropin-releasing hormone; RCTs: Randomized controlled trials; ET: Embryo transfer; IVF: In vitro fertilization; ICSI: Intracytoplasmic sperm injection; OHSS: Ovarian hyperstimulation syndrome; ORs: Odds ratios; Cls: Confidence intervals}

\section{Supplementary Information}

The online version contains supplementary material available at https://doi. org/10.1186/s12958-021-00782-5.

Additional file 1: Appendix S1. MEDLINE search strategy. Appendix S2. Full references for all trials included in the review.

Additional file 2: Figure S1. Risk of bias presented as percentages across all included studies. Figure S2. Summary of risk of bias for each trial. Figure S3. Network meta-analysis (NMA) of clinical pregnancy and miscarriage according to luteal phase support initiation strategy. Abbreviations: $\mathrm{HCG}=$ human chorionic gonadotrophin; $\mathrm{VPP}=$ vaginal progesterone pessary; $V P G=$ vaginal progesterone gel; IMP=intramuscular progesterone; $\mathrm{OP}=$ oral progesterone; $\mathrm{RP}=$ rectal progesterone. Figure S4. Global and local inconsistency tests between direct and indirect estimates in the analyses of the pregnancy outcomes. Blue alphabets represent early luteal phase supports. Yellow alphabets represent delayed luteal phase supports. $A=$ placebo; $B=$ human chorionic gonadotrophin; $C=$ vaginal progesterone pessary; $\mathrm{D}=$ vaginal progesterone gel; $\mathrm{E}=$ =intramuscular progesterone; $F=$ oral progesterone; $G=$ rectal progesterone. Figure S5. Network meta-analysis (NMA) for clinical pregnancy and miscarriage. Figure S6. The effects of adding oestrogen, HCG or GnRH agonists in the luteal phase on the pregnancy outcomes. Abbreviations: $\mathrm{GnRH}$,

gonadotropin releasing hormone; HCG, human chorionic gonadotrophin. Figure S7. The effects of luteal phase support on adverse events. Figure S8. Global and local inconsistency tests between direct and indirect estimates in the analyses of the pregnancy outcomes (regardless of the initiations of luteal phase supports). $A=$ placebo; $B=$ human chorionic gonadotrophin; $C=$ vaginal progesterone pessary; $D=$ vaginal progesterone gel; $\mathrm{E}=$ =intramuscular progesterone; $\mathrm{F}=$ oral progesterone; $\mathrm{G}=$ rectal progesterone; $\mathrm{H}=$ =vaginal progesterone pessary+ oestrogen; J=intramuscular progesterone+ oestrogen; $K=$ vaginal progesterone pessary + human chorionic gonadotrophin; L=intramuscular progesterone+ human chorionic gonadotrophin; $\mathrm{N}=$ subcutaneous progesterone; $\mathrm{O}=$ =vaginal progesterone ring. Figure S9. Comparison-adjusted funnel plot for the pregnancy outcomes. Figure S10. Network meta-analysis (NMA) for ongoing pregnancy. (A) including studies since 2010, (B) including articles using long gonadotropin releasing hormone agonist protocol.

Additional file 3: Table S1. Characteristics of the included studies in the systematic review. Abbreviations: NS, not stated; IVF, in vitro fertilization; ICSI, intracytoplasmic sperm injection; $\mathrm{GnRH}$, gonadotropin releasing hormone; $\mathrm{HCG}$, human chorionic gonadotrophin; $\mathrm{FSH}$, follicle stimulating hormone; LH, luteinizing hormone; hMG, human menopausal gonadotropin; ET, embryo transfer; PD, pituitary desensitisation; SC, subcutaneous; IM, intramuscular; ART, assisted reproductive technology; BMI, body mass index; OHSS, ovarian hyperstimulation syndrome. 


\section{Acknowledgments}

We thank Aghahosseini Marzieh, Bergh Christina, Chi HB, Monique H. Mochtar, Nasrin Saharkhiz for providing unpublished data in this review and Mohamad E GHanem, Juan A Garcia-Velasco for revising the manuscript.

\section{Authors' contributions}

PZ designed the study, conducted literature searches, selected articles for inclusion, and drafted the first version of the manuscript. HLW selected articles for inclusion, extracted data, planned the statistical analyses and drafted the first version of the manuscript. SSW and XNL extracted data and assessed study quality. SYZ performed all statistical analyses. All authors contributed to the revision of the first draft of the manuscript, critically checked its content, and approved its final version.

\section{Funding}

This work was supported by the National Natural Science Foundation of China grant (81671435).

\section{Availability of data and materials}

The current study was based on the results of relevant published studies.

\section{Declarations}

\section{Ethics approval and consent to participate}

Not applicable.

\section{Consent for publication}

Not applicable.

\section{Competing interests}

The authors declare that they have no competing interests.

\section{Author details}

${ }^{1}$ Department of Obstetrics and Gynaecology, Hangzhou Women's Hospital, No. 369 Kun Peng Road, Hangzhou 310008, Zhejiang, China. ${ }^{2}$ Assisted Reproduction Unit, Department of Obstetrics and Gynecology, Sir Run Run Shaw Hospital, Zhejiang University School of Medicine, No. 3 Qingchun East Road, Hangzhou 310016, China.

Received: 27 January 2021 Accepted: 12 June 2021

Published online: 06 July 2021

\section{References}

1. de Mouzon J, Chambers GM, Zegers-Hochschild F, Mansour R, Ishihara O, Banker $\mathrm{M}$, et al. International Committee for Monitoring Assisted Reproductive Technologies world report: assisted reproductive technology 2012. Hum Reprod. 2020;35(8):1900-13. https://doi.org/10.1093/humrep/ deaa090.

2. Fatemi HM. The luteal phase after 3 decades of IVF: what do we know? Reprod BioMed Online. 2009;19:4331.

3. van der Linden M, Buckingham K, Farquhar C, Kremer JA, Metwally M. Luteal phase support for assisted reproduction cycles. Cochrane Database Syst Rev. 2015;7:CD009154.

4. Jiang $\mathrm{CL}$, Hu DL, Peng HY, Wang H, Zhang CJ. Effects of human chorionic gonadotropin combined progesterone as luteal support on the outcome of IVF-ET in patients with low OHSS risk. J Yunyang Med College. 2009;28:357-62.

5. Lan MA, Chan Y, Chen JS. The influence of pregnancy outcomes with estradiol valerate in addition during luteal phase in IVF-ET Cycles. J Kunming Med Univ. 2013;11:93-6.

6. Kutlusoy F, Guler I, Erdem M, Erdem A, Bozkurt N, Biberoglu EH, et al. Luteal phase support with estrogen in addition to progesterone increases pregnancy rates in in vitro fertilization cycles with poor response to gonadotropins. Gynecol Endocrinol. 2014;30(5):363-6. https://doi.org/10.31 09/09513590.2014.887065.

7. Kwon SK, Kim CH, Lee KH, Jeon IK, Ahn JW, Kim SH, et al. Luteal estradiol supplementation in gonadotropin-releasing hormone antagonist cycles for infertile patients in vitro fertilization. Clin Exp Reprod Med. 2013;40(3):131-4. https://doi.org/10.5653/cerm.2013.40.3.131

8. Moini A, Zafarani F, Eslami B, Sadeghi M, Kamyabi Z, Jahangiri N. Comparing intramuscular progesterone, vaginal progesterone and 17 alpha-
Hydroxyprogestrone caproate in IVF and ICSI cycle. Iran J Reprod Med. 2011;9(2):119-24.

9. Soon N, Sang Hoon L, Do Whan B. A comparative study on clinical effectiveness of human chorionic gonadotropin and progesterone on luteal support in Controlled Ovarian Hyperstimulatian far IVF. Korea J Fertil Steril. 1994;21:233-40

10. Razieh DF, Maryam AR, Nasim T. Beneficial effect of luteal-phase gonadotropin-releasing hormone agonist administration on implantation rate after intracytoplasmic sperm injection. Taiwan J Obstet Gynecol. 2009; 48(3):245-8. https://doi.org/10.1016/S1028-4559(09)60297-7.

11. Wang XX, Yi XF, Fang YY, Zhao DN, Ye YX. The influence of pregnancy outcomes with oestradiol valeratein addition during luteal phase in IVF-ET cycles. Chin J Pract Gynecol Obstet. 2009;25:938-40.

12. Zhang H, Wang W, Ma W, Cai Q, Liu Y. The influence of pregnancy outcomes with oestradiol valerate in addition to progesterone for luteal supplementation in IVF-ET cycles. Matern Child Health Care China. 2008;29: 4163-5.

13. Chavez FCP, Delgadillo JCB, Rueda SSO, Villa GB, Acosta SV, Solis VS, et al. Estrogen role in the luteal phase support in in vitro fertilization with embryo transfer cycles. Ginecol Obstet Mex. 2004;72:645-55.

14. Miller CE, Zbella E, Webster BW, Doody KJ, Bush MR, Collins MG. Clinical comparison of ovarian stimulation and luteal support agents in patients undergoing GnRH antagonist IVF cycles. J Reprod Med. 2013;58(3-4):153-60.

15. Tay PY, Lenton EA. Inhibition of progesterone secretion by oestradiol administered in the luteal phase of assisted conception cycles. Med J Malaysia. 2003;58(2):187-95.

16. Benmachiche A, Benbouhedja S, Zoghmar A, Boularak A, Humaidan P. Impact of mid-luteal phase $\mathrm{GnRH}$ agonist administration on reproductive outcomes in $\mathrm{GnRH}$ agonist-triggered cycles: a randomized controlled trial. Front Endocrinol. 2017:8:124. https://doi.org/10.3389/fendo.2017.00124.

17. Chi H, Li R, Qiao J, Chen X, Wang X, Hao G, et al. Vaginal progesterone gel is non-inferior to intramuscular progesterone in efficacy with acceptable tolerability for luteal phase support: a prospective, randomized, multicenter study in China. Eur J Obstet Gynecol Reprod Biol. 2019;237:100-5. https:// doi.org/10.1016/j.ejogrb.2019.04.012.

18. Gao J, Gu F, Miao BY, Chen MH, Zhou CQ, Xu YW. Effect of the initiation of progesterone supplementation in in vitro fertilization-embryo transfer outcomes: a prospective randomized controlled trial. Fertil Steril. 2018, 109(1):97-103. https://doi.org/10.1016/j.fertnstert.2017.09.033.

19. Ghanem ME, Bedairy MH, Shaaban A, Albahlol IA. Does the time of starting progesterone luteal support affect embryo transfer in long agonist protocol downregulated ICSI cycles? A Randomized Controlled Trial. Reprod Sci. 2020;28(3):897-903. https://doi.org/10.1007/s43032-020-00309-0.

20. Griesinger G, Blockeel C, Sukhikh G, Patki A, Dhorepatil B, Yang DZ, et al. Oral dydrogesterone versus intravaginal micronized progesterone gel for luteal phase support in IVF: a randomized clinical trial. Hum Reprod. 2018; 10:1-10.

21. Ismail Madkour WA, Noah B, Abdel Hamid AMS, Zaheer H, Al-Bahr A, Shaeer $M$, et al. Luteal phase support with estradiol and progesterone versus progesterone alone in GnRH antagonist ICSI cycles: a randomized controlled study. Hum Fertil. 2016;19(2):142-9. https://doi.org/10.1080/1464 7273.2016.1200145.

22. Kapur A, Prasad S, Kumar A. Is luteal phase estradiol supplementation beneficial in long agonist IVF-ET cycles? First prospective randomised controlled study from indian subcontinent. J Clin Diagn Res. 2018;12:QC01-3.

23. Khrouf M, Slimani S, Khrouf MR, Braham M, Bouyahia M, Berjeb KK, et al. Progesterone for luteal phase support in in vitro fertilization: comparison of vaginal and rectal pessaries to vaginal capsules: a randomized controlled study. Clin Med Insights Womens Health. 2016;9:43-7. https://doi.org/10.413 7/CMWH.S32156.

24. Michnova L, Dostal J, Kudela M, Hamal P, Langova K. Vaginal use of micronized progesterone for luteal support. A randomized study comparing Utrogestan ${ }^{\oplus}$ and Crinone ${ }^{\circledast}$ 8. Biomed Papers Med Faculty Univ Palacky Olomouc Czechoslovakia. 2017;161(1):86-91. https://doi.org/10.5507/bp.2017.007.

25. Saharkhiz N, Zamaniyan M, Salehpour S, Zadehmodarres SH, Hoseini S, Cheraghi $L$, et al. A comparative study of dydrogesterone and micronized progesterone for luteal phase support during in vitro fertilization (IVF) cycles. Int J Reprod Biomed. 2017;15:45.

26. Samsami A, Zarei A, Shahrivar S. Effects of estradiol injection on outcome of in-vitro fertilization: a randomized, double-blind, placebo controlled trial. Clin Exp Obstet Gynecol. 2016;43(6):875-9. 
27. Tomic V, Tomic J, Klaic DZ, Kasum M, Kuna K. Oral dydrogesterone versus vaginal progesterone gel in the luteal phase support: randomized controlled trial. Eur J Obstet Gynecol Reprod Biol. 2015;186:49-53. https:// doi.org/10.1016/j.ejogrb.2014.11.002.

28. Tournaye H, Sukhikh GT, Kahler E, Griesinger G. A phase III randomized controlled trial comparing the efficacy, safety and tolerability of oral dydrogesterone versus micronized vaginal progesterone for luteal support in in vitro fertilization. Hum Reprod. 2017;32(5):1019-27. https://doi.org/10.1 093/humrep/dex023.

29. Zafardoust S, Jeddi-Tehrani M, Akhondi MM, Sadeghi MR, Kamali K, Mokhtar S, et al. Effect of Administration of Single Dose GnRH agonist in luteal phase on outcome of ICSI-ET cycles in women with previous history of IVF/ICS failure: a randomized controlled trial. J Reprod Infertil. 2015;16(2):96-101.

30. Zargar M, Saadati N, Ejtahed MS. Comparison the effectiveness of oral dydrogesterone, vaginal progesterone suppository and progesterone ampule for luteal phase support on pregnancy rate during ART cycles. Int J Pharma Res Allied Sci. 2016;5:229-36.

31. Fusi FM, Brigante CM, Zanga L, Mignini Renzini M, Bosisio C, Fadini R. GnRH agonists to sustain the luteal phase in antagonist IVF cycles: a randomized prospective trial. Reprod Biol Endocrinol. 2019;17(1):103. https://doi.org/1 0.1186/s12958-019-0543-2.

32. Barbosa MWP, Valadares NPB, Barbosa ACP, Amaral AS, Iglesias JR, Nastri CO, et al. Oral dydrogesterone vs. vaginal progesterone capsules for luteal-phase support in women undergoing embryo transfer: a systematic review and meta-analysis. JBRA Assist Reprod. 2018;22(2):148-56. https://doi.org/10.593 5/1518-0557.20180018.

33. Doblinger J, Cometti B, Trevisan S, Griesinger G. Subcutaneous progesterone is effective and safe for luteal phase support in IVF: an individual patient data meta-analysis of the phase III trials. PLoS One. 2016;11(3):e0151388. https://doi.org/10.1371/journal.pone.0151388.

34. Martins WP, Ferriani RA, Navarro PA, Nastri CO. GnRH agonist during luteal phase in women undergoing assisted reproductive techniques: systematic review and meta-analysis of randomized controlled trials. Ultrasound Obstet Gynecol. 2016;47(2):144-51. https://doi.org/10.1002/uog.14874.

35. Ma X, Du W, Hu J, Yang Y, Zhang X. Effect of gonadotrophin-releasing hormone agonist addition for luteal support on pregnancy outcome in vitro fertilization/intracytoplasmic sperm injection cycles: a meta-analysis based on randomized controlled trials. Gynecol Obstet Investig. 2020;85(1): 13-25. https://doi.org/10.1159/000501204.

36. Connell MT, Szatkowski JM, Terry N, DeCherney AH, Propst AM, Hill MJ. Timing luteal support in assisted reproductive technology: a systematic review. Fertil Steril. 2015;103:939-46.e3.

37. Hutton B, Salanti G, Caldwell DM, Chaimani A, Schmid CH, Cameron C, et al. The PRISMA extension statement for reporting of systematic reviews incorporating network meta-analyses of health care interventions: checklist and explanations. Ann Intern Med. 2015;162(11):777-84. https://doi.org/10. 7326/M14-2385

38. Higgins JP, Altman DG, Gøtzsche PC, Jüni $P$, Moher $D$, Oxman AD, et al. The Cochrane Collaboration's tool for assessing risk of bias in randomised trials. BMJ. 2011;343:d5928.

39. Rodriguez-Pezino J, Saucedo-de la Llata E, Batiza-Resendiz V, Galache-Vega P, Santos-Haliscak R, Hernandez-Ayup S, et al. Vaginal progesterone in assisted reproduction. Hum Reprod. 2004;19(Suppl 1):i51.

40. Ng EHY, Chan CCW, Tang OS, Ho PC. A randomized comparison of side effects and patient convenience between Cyclogest suppositories and Endometrin tablets used for luteal phase support in IVF treatment. Eur J Obstet Gynecol Reprod Biol. 2007;131(2):182-8. https://doi.org/10.1016/j. ejogrb.2006.07.023

41. Dal Prato L, Bianchi L, Cattoli M, Tarozzi N, Flamigni C, Borini A. Vaginal gel versus intramuscular progesterone for luteal phase supplementation: a prospective randomized trial. Reprod BioMed Online. 2008;16(3):361-7. https://doi.org/10.1016/S1472-6483(10)60597-4

42. Doody KJ, Schnell VL, Foulk RA, Miller CE, Kolb BA, Blake EJ, et al. Endometrin for luteal phase support in a randomized, controlled, openlabel, prospective in-vitro fertilization trial using a combination of Menopur and Bravelle for controlled ovarian hyperstimulation. Fertil Steril. 2009;91(4): 1012-7. https://doi.org/10.1016/j.fertnstert.2008.01.069.

43. Higgins JP, Thompson SG, Deeks JJ, Altman DG. Measuring inconsistency in meta-analyses. BMJ. 2003;327(7414):557-60. https://doi.org/10.1136/bmj.327. 7414.557.
44. Chaimani A, Higgins JP, Mavridis D, Spyridonos P, Salanti G. Graphical tools for network meta-analysis in STATA. PLoS One. 2013;8(10):e76654. https:// doi.org/10.1371/journal.pone.0076654.

45. Salanti G. Indirect and mixed-treatment comparison, network, or multipletreatments meta-analysis: many names, many benefits, many concerns for the next generation evidence synthesis tool. Res Synth Methods. 2012;3(2): 80-97. https://doi.org/10.1002/jssm.1037.

46. Salanti G, Del Giovane C, Chaimani A, Caldwell DM, Higgins JP. Evaluating the quality of evidence from a network meta-analysis. PLoS One. 2014;9(7): e99682. https://doi.org/10.1371/journal.pone.0099682.

47. White IR. Network meta-analysis. Stata J. 2015;15(4):951-85. https://doi.org/1 $0.1177 / 1536867 \times 1501500403$.

48. Guyatt GH, Oxman AD, Schunemann HJ, Tugwell P, Knottnerus A. GRADE guidelines: a new series of articles in the journal of clinical epidemiology. J Clin Epidemiol. 2011;64(4):380-2. https://doi.org/10.1016/j.jclinepi.2010.09.011.

49. Feichtinger $M$, Hejek J, Kemter $P$, Feichtinger $W$. Effect of luteal phase support comparing early (day 1) and late (day 4) initiation with pregnancy rates. J Reprod Med Endocrinol. 2011;8:288-90.

50. Williams SC, Oehninger S, Gibbons WE, Van Cleave WC, Muasher SJ. Delaying the initiation of progesterone supplementation results in decreased pregnancy rates after in vitro fertilization: a randomized, prospective study. Fertil Steril. 2001;76(6):1140-3. https://doi.org/10.1016/ S0015-0282(01)02914-4.

51. Huang N, Situ B, Chen X, Liu J, Yan P, Kang X, et al. Meta-analysis of estradiol for luteal phase support in in vitro fertilization/intracytoplasmic sperm injection. Fertil Steril. 2015;103:367-73.e5.

52. Kyrou D, Kolibianakis EM, Fatemi HM, Tarlatzi TB, Devroey P, Tarlatzis BC. Increased live birth rates with GnRH agonist addition for luteal support in ICSI/IVF cycles: a systematic review and meta-analysis. Hum Reprod Update. 2011;17(6):734-40. https://doi.org/10.1093/humupd/dmr029.

\section{Publisher's Note}

Springer Nature remains neutral with regard to jurisdictional claims in published maps and institutional affiliations.

Ready to submit your research? Choose BMC and benefit from

- fast, convenient online submission

- thorough peer review by experienced researchers in your field

- rapid publication on acceptance

- support for research data, including large and complex data types

- gold Open Access which fosters wider collaboration and increased citations

- maximum visibility for your research: over $100 \mathrm{M}$ website views per year

At BMC, research is always in progress.

Learn more biomedcentral.com/submissions 